\title{
HACIA UN NUEVO MODELO DE ATENCIÓN PRIMARIA EN SALUD EVALUACIÓN DEL PROYECTO DE SALUD FAMILIAR ANCORA UC*
}

\author{
K. Püschel, A. Téllez, J. Montero, A. Brunner, B. Peñaloza, \\ M. P. Rojas, F. Poblete, T. Pantoja \\ P. Universidad Católica de Chile
}

\begin{abstract}
Resumen: Este estudio presenta una evaluación de diseño transversal que compara la efectividad clínica, satisfacción usuaria e impacto económico de la red de centros de salud
\end{abstract}

Klaus Püschel. Médico, P. Universidad Católica de Chile (PUC). Magíster en Salud Pública, Universidad de Washington, Profesor Asociado, Escuela de Medicina, PUC. Dirección electrónica: kpuschel@med.puc.cl.

Álvaro Téllez. Médico, P. Universidad Católica de Chile (PUC). Magíster en Salud Pública, Universidad de Chile. Profesor Asociado Escuela de Medicina, PUC. Dirección electrónica: atellez@med.puc.cl.

Joaquín Montero. Médico, P. Universidad Católica de Chile (PUC). Magíster en Salud Pública, Universidad de North Carolina, Profesor titular Escuela de Medicina, PUC. Dirección electrónica: jmontero@med.puc.cl.

Astrid Brunner. Enfermera Matrona, P. Universidad Católica de Chile. Directora Red Centros de Salud Familiar, PUC. Dirección electrónica: abrunner@med.puc.cl.

* El estudio recibió financiamiento parcial de la Corporación Nacional de investigación de Ciencia y Tecnología (CONICYT) mediante el proyecto FONIS SA07I20032 y de la Facultad de Medicina de la Pontifica Universidad Católica de Chile, que encargó una evaluación externa de resolutividad a la Consultora Epidemiología y Gestión. 
familiar Ancora UC con la red de centros municipales de las comunas de Puente Alto y La Pintana, donde los centros Ancora están ubicados. El estudio muestra que Ancora UC logró una mayor efectividad clínica expresada en reducciones del $25 \%$ de derivaciones a especialidades secundarias, $70 \%$ de consultas de urgencia y $30 \%$ de hospitalizaciones respecto a los centros comparadores. Las diferencias se mantuvieron al ajustar por variables sociodemográficas. Estos efectos se asociaron a una alta satisfacción usuaria y representaron un ahorro estimado de 1.690 millones de pesos para el sistema de salud el año 2010. Los resultados son consistentes con experiencias similares desarrolladas en Canadá, Inglaterra y Brasil y enfatizan el impacto positivo de una atención primaria integral y resolutiva.

Palabras clave: atención primaria, evaluación, políticas de salud, salud familiar.

Recibido: septiembre 2012; aceptado: marzo 2013.

\section{TOWARDS A NEW MODEL OF PRIMARY CARE NETWORK: ASESSMENT OF THE FAMILY HEALTH CARE NETWORK, ANCORA, UC}

Abstract: In this study a cross sectional evaluation was conducted to compare clinical effectiveness, user satisfaction and economic effects of a university based family health care network, Ancora UC, with the municipal primary care network from the counties where the Ancora centers are located. The study shows that Ancora UC obtained higher clinical effectiveness expressed in reductions of $20 \%$ of referrals to secondary care, $70 \%$ in emergency room visits and $30 \%$ of hospitalizations compared to the

Blanca Peñaloza. Médico, P. Universidad Católica de Chile (PUC). Magíster en Ciencia y Gestión en Salud, Universita Luigi Bocconi, Italia. Magister en Tecnologías en Salud, Universidad de Birmingham, Reino Unido. Profesora asistente, Escuela de Medicina, PUC. Dirección electrónica: bpenalo@med.puc.cl.

María Paulina Rojas. Médico, P. Universidad Católica de Chile (PUC).Profesora asociada, Escuela de Medicina, PUC. Dirección electrónica: marojas@med.puc.cl.

Fernando Poblete. Médico, P. Universidad Católica de Chile (PUC). Magíster en Salud Pública, Universidad de Harvard, Profesor Asistente, Escuela de Medicina, PUC. Dirección electrónica: fpoblete@med.puc.cl.

Tomás Pantoja. Médico, P. Universidad Católica de Chile (PUC). Magíster en Ciencias y Gestión en Salud, Imperial College (Gr. Br.), Profesor Asociado, Escuela de Medicina, PUC. Dirección electrónica: tpantoja@med.puc.cl. 
municipal health care network. The differences remained significant after adjusting for socio-demographic variables. The outcomes are associated with a high level of user's satisfaction and represented an estimated saving of 1,690 millions of Chilean pesos in 2010. The results are consistent with the ones observed in similar experiences in Canada, England and Brazil and highlight the positive impact of an integral primary care model.

Keywords: primary care, evaluation, health policy, family health.

Received: September 2012; accepted: March 2013.

\section{Introducción}

Trad radicionalmente, el nivel primario de atención ha sido identificado como el ámbito de primer contacto de la población con el sistema de salud. En este nivel de atención se desarrolla la atención primaria de salud (APS) que ha sido históricamente entendida como un escenario de atención básica de baja complejidad técnica, alta cobertura poblacional y fuerte vinculación comunitaria (Declaración Alma Ata, 1978).

Este concepto de APS básica comenzó a variar significativamente desde mediados de los años 90 debido a la evidencia emergente respecto del efecto de diferentes modelos de APS en el sistema de salud de países de alto desarrollo socioeconómico. En un clásico estudio internacional liderado por Starfield (Starfield y Shi, 2002), que incluyó trece países desarrollados pertenecientes a la Organización para la Cooperación y Desarrollo (OCDE), se observó que países con una APS más "fuerte" obtenían mejores indicadores de eficiencia y efectividad que países con una APS más "débil". Países como Inglaterra, Dinamarca o España, calificados con una APS fuerte, tenían mejores indicadores de salud tales como mortalidad infantil o años de vida potencialmente perdidos en comparación con países con una APS débil como EE. UU. o Alemania. Además, el estudio demostró que países con una APS más desarrollada obtenían mejores niveles de satisfacción usuaria y menores gastos en salud que países con una APS menos avanzada (Starfield et al., 2005).

Los resultados obtenidos por el grupo de Starfield se han repetido en forma consistente en estudios posteriores. En una revisión sistemática de la literatura desarrollada por Kringos et al. (2010) se identificaron 85 estudios metodológicamente adecuados y se observó 
una robusta asociación entre desarrollo de APS e indicadores globales de salud en países con nivel de desarrollo económico medio y alto.

A partir de esa evidencia, se realizaron investigaciones focalizadas en variables intermedias que, a nivel local, pudieran explicar las diferencias observadas en estudios de comparación internacional. Variables tales como tasa de derivación y consulta a especialidades, consulta en servicios de urgencia y tasa de hospitalizaciones fueron las que demostraron mayor asociación con los macroindicadores poblacionales de morbilidad y mortalidad mencionados (Starfield, 2012). Al analizar con detalle los factores asociados a estas variables intermedias puede observarse que países con APS más avanzada cuentan con equipos de profesionales con mayor formación técnica en APS, mayor tecnología disponible en sus centros de atención y una organización de trabajo en equipo que combina atención clínica efectiva con trabajo centrado en la familia y la comunidad (Pasarín et al., 2007). Modelos de APS con estas características se asocian a estrategias de atención más integrales, menos redundantes y más eficientes (Starfield, 2012).

Los profundos cambios ocurridos en las últimas dos décadas a partir de la evidencia empírica descrita han provocado un cambio en la percepción del concepto de APS. Éste ha experimentado un "viraje" desde el tradicional concepto de atención "básica" hacia uno de atención "compleja" promovido por la Organización Mundial de la Salud (OMS), que incluye desarrollo profesional, utilización de tecnología y trabajo interdisciplinario que debiera ser adoptada por todos los países (OMS, 2008).

En este estudio se realiza una evaluación del proyecto piloto Red Ancora UC, cuyo propósito es contribuir al mejoramiento de la atención de salud de poblaciones vulnerables. El proyecto ha sido desarrollado por la Facultad de Medicina de la Pontificia Universidad Católica (PUC) (gracias a una donación recibida el año 2003), en colaboración con el Ministerio de Salud (Minsal), el Servicio de Salud Metropolitano Sur Oriente (SSMSO) y las Municipalidades de Puente Alto y La Pintana.

Ancora UC recoge la experiencia de salud primaria chilena pero introduce significativas innovaciones en cuatro áreas esenciales. En el área de gestión, el proyecto introduce un nuevo modelo mixto, públicoprivado, para el financiamiento y la prestación de servicios. En el área de recursos humanos, el proyecto desarrolla un modelo de formación de profesionales de salud con competencias específicas ausentes en la APS actual. En el área tecnológica, el proyecto introduce nuevas tecnologías 
de apoyo clínico y sistemas de registro informático no disponibles en la actual APS chilena. Por último, en el área de modelo de atención de salud, el proyecto profundiza el enfoque familiar de la APS aplicándolo a necesidades clínicas y de salud específicas. En este estudio se analiza la implementación de estas innovaciones y el impacto de ellas desde la perspectiva económica, poblacional y de efectividad clínica.

\section{Desafíos de atención primaria para Chile}

Chile, al igual que muchos países emergentes, ha experimentado un cambio radical en su perfil epidemiológico en los últimos 30 años. El país ha pasado de un escenario de predominio de enfermedades agudas y problemas de salud materno-infantiles a uno de claro predominio de enfermedades crónicas y de salud mental. Las enfermedades cardiovasculares, el cáncer, las enfermedades respiratorias, los trastornos depresivos y problemas derivados del consumo excesivo de alcohol, representan hoy alrededor de dos tercios de la carga de morbi-mortalidad de la población (Ministerio de Salud, 2007).

El nuevo perfil epidemiológico presenta nuevos desafíos que no han sido bien resueltos por el sistema de salud chileno. Los estudios poblacionales en Chile han demostrado una alta prevalencia de factores de riesgo de enfermedades crónicas y un muy bajo porcentaje de control de las mismas. La última Encuesta Nacional de Salud (Ministerio de Salud, 2011) mostró que sólo $16,5 \%$ de las personas con hipertensión arterial y $34,3 \%$ de las personas con diabetes mellitus se encontraban bien compensadas de su enfermedad. Si a esta realidad se agrega la alta prevalencia de la población con dislipidemia (38\%), tabaquismo $(40,6 \%)$, obesidad $(25,1 \%)$ y sedentarismo $(88,6 \%)$, se entiende que exista un $18 \%$ de la población con riesgo cardiovascular alto. Este porcentaje es más alto aún en la población de bajo nivel socioeconómico, en donde alrededor de un tercio de la misma se encuentra en esta condición.

La realidad epidemiológica descrita enfrenta al sistema de salud chileno con un nuevo escenario que requiere una adaptación de su modelo, tal como ha sucedido en otros países de alto nivel de desarrollo. Esta adaptación debiera considerar el fortalecimiento de la APS chilena, necesidad que ha sido claramente reconocida por autoridades políticas y académicas (Cámara de Diputados de Chile, 2012). Una mala adaptación puede generar dificultades que se expresan habitualmente 
en derivaciones inapropiadas o tardías de pacientes desde la APS a los niveles secundarios o terciarios, lo que incrementa las listas de espera generando ineficiencias y mayor riesgo en salud. Otra consecuencia de una mala adaptación del sistema a los cambios de perfil epidemiológico se expresa en una sobrecarga inapropiada y riesgosa de los sistemas de atención de urgencia y de los ingresos hospitalarios (Macinko et al., 2009; Kringos et al., 2010).

La reforma de salud implementada en Chile, si bien contemplaba originalmente un fortalecimiento de la atención primaria y un cambio en el modelo de atención, desde uno esencialmente curativo a otro que incorporara con mayor fuerza la prevención y la integralidad en la atención, no ha sido fiel a estos principios (Asociación Chilena de $\mathrm{Mu}-$ nicipalidades, 2009; Montero et al., 2010; Baas, 2012). La baja incorporación de la APS en la implementación de la reforma de salud ha tenido al menos tres consecuencias severas para el sistema y su población. En primer término, una ausencia de mecanismos de control en la generación de listas de espera en especialidades. De esta forma, la incidencia de derivaciones se ha mantenido o aumentado desde el inicio de la reforma, según el Departamento de Estadísticas e Información de Salud (DEIS), (DEIS, 2009 y 2012). En segundo lugar, una sobrecarga en la atención de los servicios de urgencia (DEIS, 2009; DEIS, 2012). Por último, una fragmentación de la atención a las personas al separar la atención de problemas de salud altamente asociados entre sí, como hipertensión arterial, diabetes mellitus y trastornos depresivos (Stange, 2009; Lin et al., 2012). Esta fragmentación reduce la integralidad e incrementa la ineficiencia en la atención de usuarios de la APS (Starfield, 2012). De acuerdo a la evidencia presentada previamente, ambos factores, sobrecarga de atención en urgencia y fragmentación de la atención, son una señal de un sistema de salud con una APS débil.

El proyecto Ancora UC se planteó como una estrategia innovadora para contribuir a enfrentar los problemas descritos de la realidad de la APS chilena. Lo que sigue de este artículo describe el proceso y los resultados de este proyecto y se divide así: en primer término, se describen los elementos esenciales del diseño e implementación del proyecto Ancora UC (sección 3). En segundo lugar, se presenta la metodología de evaluación del proyecto en tres dimensiones: satisfacción usuaria, efectividad clínica y balance económico (sección 4). Luego se presentan los resultados del proyecto en las tres dimensiones mencionadas (sección 5). En la última sección se discuten los resultados del proyecto a la luz de la experiencia internacional y sus implicancias en las políticas de salud del país. 


\section{Diseño e implementación}

El objetivo general del proyecto fue contribuir al desarrollo de la atención primaria chilena mediante la implementación de un modelo innovador de salud basado en enfoque familiar, gestión informatizada, efectividad clínica, y capacidad formativa. Al cabo de cuatro años de iniciado el proyecto, se construyeron e implementaron tres nuevos centros de salud familiar, dos en la comuna de Puente Alto, denominados Madre Teresa de Calcuta (MTC) y San Alberto Hurtado (SAH), y uno en La Pintana, denominado Juan Pablo II (JPII).

\section{Inscripción de la población}

Los criterios de inscripción de la población en los centros Ancora UC quedaron estipulados en el convenio firmado entre la PUC, el SSMSO y el Minsal. Según el convenio, el mecanismo de inscripción debía ser idéntico al existente en todos los centros municipales del país. La inscripción sólo requería la voluntad del usuario y pertenecer al Fondo Nacional de Salud (Fonasa). Al igual que en los centros municipales del país, los datos requeridos para inscribirse en Ancora UC fueron los de identificación y fecha de nacimiento del interesado. De acuerdo a la legislación vigente, los usuarios tienen libertad para elegir el centro donde quieren inscribirse. El proceso de inscripción no discrimina en base a ningún antecedente demográfico, médico o social. De esta forma, no es posible negar la inscripción a un centro de un interesado perteneciente a Fonasa.

La ubicación geográfica de los centros quedó definida de común acuerdo con las autoridades municipales de Puente Alto y La Pintana. Ellas definieron las áreas geográficas de su interés para instalar los centros Ancora UC en base a vacíos de cobertura y donaron, en todos los casos, los terrenos para la construcción de los centros. De esta forma, el proceso de inscripción no incluyó ningún mecanismo de selección adversa de la población. Tres factores findamentales impedían que se diera una selección adversa de la población. En primer lugar, hacerlo era ilegal; en segundo lugar, el perfil de salud de la población al momento de la inscripción no era conocido y, en tercer lugar, los propios municipios definieron los lugares prioritarios de instalación de los centros. Finalmente, cualquier mecanismo de selección adversa implicaba una contradicción interna con el proyecto voluntario de la 
PUC, cuya razón esencial de desarrollo era precisamente el servicio a poblaciones vulnerables.

\section{Financiamiento}

El financiamiento de los nuevos centros tuvo dos componentes esenciales. El primero fue la donación recibida, con lo que se financió la construcción y la implementación física de los nuevos centros. El segundo componente se vinculó a la operación de los centros. Para ello, se firmó un convenio entre la PUC, el SSMSO y el Minsal. A través de este convenio, la universidad asumía el compromiso de entregar un conjunto de prestaciones a la población beneficiaria de Fonasa inscrita en los centros Ancora UC. Por su parte, Fonasa debía aportar un monto mensual per cápita (por persona inscrita). Este convenio aseguraba que, como ocurre en todos los centros de atención primaria del país, las prestaciones fueran totalmente gratuitas para los usuarios inscritos. El compromiso asistencial asumido por los centros Ancora UC contempló una canasta de prestaciones superior al exigido a la atención primaria. El financiamiento, sin embargo, era equivalente al de la atención primaria chilena.

El Gráfico $\mathrm{N}^{\circ} 1$ muestra los ingresos, egresos y márgenes obtenidos por el proyecto Ancora UC para los años 2005-2012. En el gráfico

GRÁFICO No 1: INGRESO, GASTO Y MARGEN ANUAL RED ANCORA UC AÑOS 2005-2012

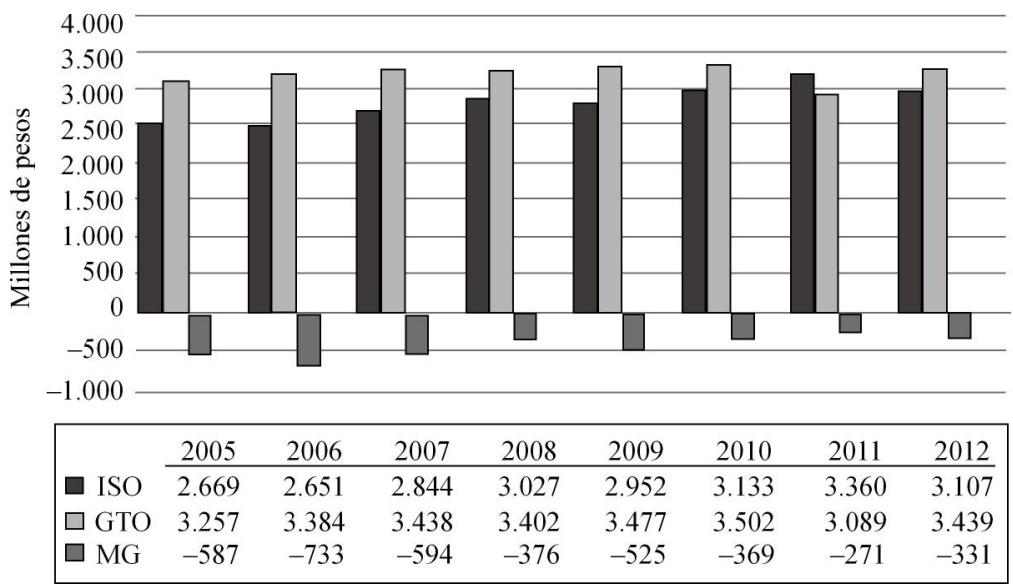

ISO: ingresos GTO: gastos MG: margen. 
puede observarse que el gasto de los centros ha tendido a reducirse en el tiempo, lo que ha permitido que el margen operacional negativo tienda a la disminución y que actualmente se encuentre cercano al 11\% de los ingresos. El Gráfico $\mathrm{N}^{\circ} 2$ muestra un análisis comparativo del reajuste anual de los ingresos per cápita percibidos por la APS municipal vs. el percibido por Ancora UC para el período 2005-2012. Este gráfico muestra que al inicio del proyecto los centros Ancora UC recibieron un monto per cápita equivalente al de la APS municipal. Sin embargo, a lo largo del tiempo los centros Ancora UC han recibido menos ingresos que los centros municipales debido esencialmente a un menor reajuste.

\section{Modelo de salud}

El modelo de salud de los centros Ancora UC consideró cuatro ejes esenciales: equipos interdisciplinarios con enfoque familiar, ges-

\section{GRÁFICO N²: REAJUSTE ANUAL, BASAL PER CÁPITA RECIBIDO DE MUNICIPIOS Y ANCORA UC AÑOS 2005-2012}

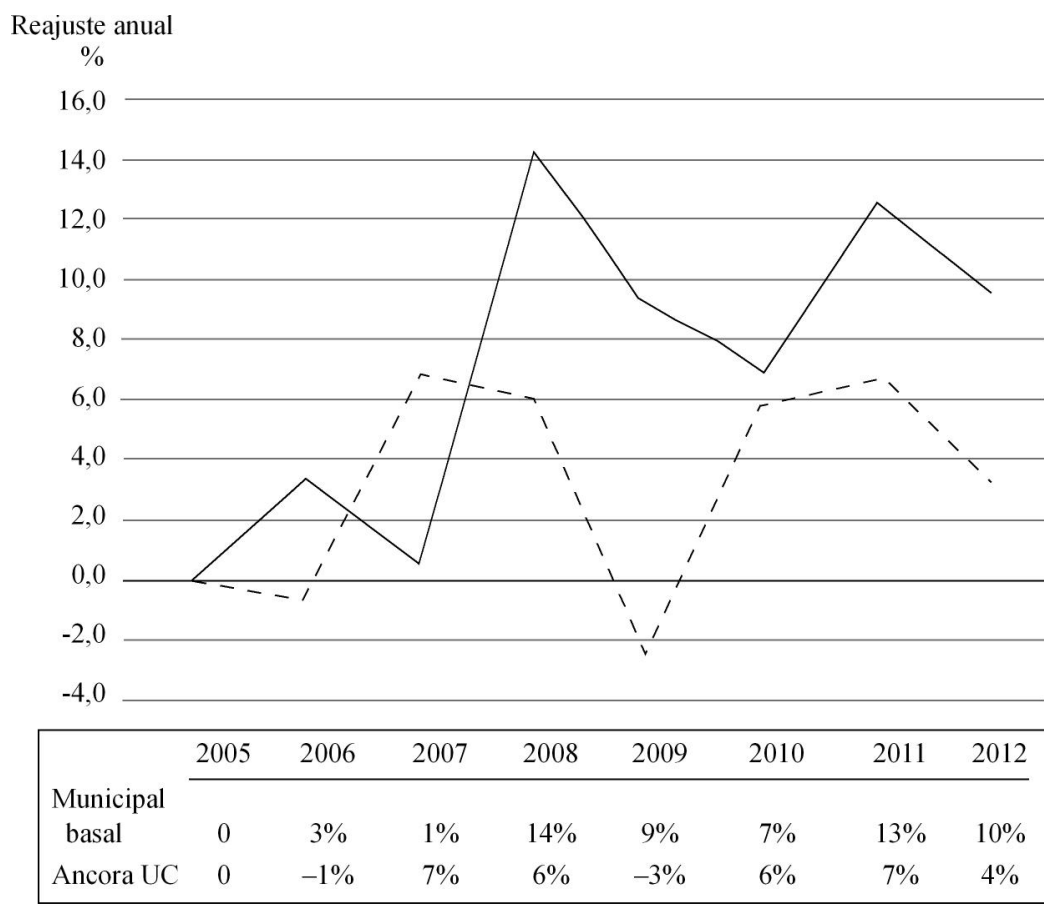

— Municipal basal. _ - - Ancora UC. 
tión informatizada, efectividad clínica y capacidad formativa. Estos ejes se definieron en base a las fortalezas existentes en la APS chilena y en base a la información emergente sobre los componentes de una APS fuerte.

\section{Equipos interdisciplinarios con enfoque familiar}

El desarrollo de esta dimensión implicó formar equipos de cabecera que asumieran el cuidado de la salud en tres niveles: individual, familiar y comunitario. Para ello, la atención fue sectorizada y, dependiendo del lugar de residencia, las familias inscritas quedaron registradas con un equipo de cabecera y un profesional preferente para la atención médica individual. La dotación de profesionales en los equipos se definió utilizando los estándares de recursos humanos disponibles al inicio del proyecto por la Organización Mundial de la Salud y el Minsal (Soto, 2003). La organización del espacio físico de los nuevos centros se diseñó considerando el perfil de equipo definido. La Tabla $\mathrm{N}^{\circ} 1$ resume los recursos humanos y físicos del proyecto.

El modelo de atención clínica contempló una estrategia segmentada para responder mejor a las necesidades cambiantes de atención de la población. Se definieron cinco modalidades de atención: atención inmediata de alto rendimiento para pacientes con enfermedades agudas, atención electiva para pacientes con problemas subagudos o enfermedades crónicas, atención familiar integral, contacto telefónico y atención domiciliaria. La intensidad del enfoque familiar utilizado fue diferente dependiendo de la modalidad de atención y se definió en base a los criterios de Doherty y Baird (1983). Según estos criterios, la aplicación del enfoque familiar es muy breve e informativa en la modalidad de atención inmediata y más profunda y terapéutica en la modalidad de atención familiar integral. Esta última se diseñó como una prestación interdisciplinaria desarrollada por duplas de profesionales de las áreas biomédica y psicosocial y estuvo dirigida a dar consejería y apoyo terapéutico a familias multiproblematizadas. El modelo de duplas se basó en el desarrollado originalmente por Hidalgo y Carrasco (2000) y buscó ampliar el espectro de atención clínica incorporando una perspectiva bio-psicosocial más consistente con la realidad de la APS. 
TABLA N ${ }^{\circ}$ 1: $\quad$ MODELO DE SALUD ANCORA UC: RECURSOS FÍSICOS, RECURSOS HUMANOS SELECCIONADOS E INNOVACIONES ESPECÍFICAS

\begin{tabular}{|c|c|}
\hline $\begin{array}{l}\text { Dimensión } \\
\text { Recursos físicos }\end{array}$ & $\begin{array}{c}\text { Indicador } \\
\text { Metraje construido }\end{array}$ \\
\hline Espacio físico total & $1.600 \mathrm{~m}^{2}$ por centro \\
\hline Espacio físico docente & $272 \mathrm{~m}^{2}$ por centro \\
\hline \multicolumn{2}{|l|}{ - Salas de supervisión en espejo } \\
\hline \multicolumn{2}{|l|}{ - Salas de discusión docente } \\
\hline Recuros humanos & Profesional por población \\
\hline Médicos & $1 / 3.333$ inscritos \\
\hline Enfermeras & $1 / 4.400$ inscritos \\
\hline Psicosociales & $1 / 5.000$ inscritos \\
\hline Técnicos paramédicos & $1 / 2.200$ inscritos \\
\hline Administrativos & $1 / 2.500$ inscritos \\
\hline \multicolumn{2}{|l|}{ Innovaciones } \\
\hline Unidad de atención inmediata & - Salas de morbilidad aguda niños y adultos \\
\hline \multirow[t]{2}{*}{ Atención familiar integral } & $\begin{array}{l}\text { - Atención familiar clínica en duplas bio- } \\
\text { psicosociales }\end{array}$ \\
\hline & $\begin{array}{l}\text { - Programa familiar de desarrollo infantil: } \\
\text { "Juguemos con nuestros hijos" (PUC/Unicef) }\end{array}$ \\
\hline Gestión informática: centro sin papel & $\begin{array}{l}\text { - Registro clínico electrónico para atención } \\
\text { primaria: OMI-AP® Stacks*. Con codifi- } \\
\text { cación internacional de atención primaria } \\
\text { (CIAP) }\end{array}$ \\
\hline \multirow[t]{3}{*}{ Procedimientos diagnósticos } & - Ecografía: abdominal y obstétrica \\
\hline & - Endoscopia digestiva alta \\
\hline & - Rectosigmoidoscopia \\
\hline Procedimientos terapéuticos & - Cirugía menor \\
\hline
\end{tabular}

* Stacks. Servicios tecnológicos, Barcelona, España. www.stacks.es/stack/ cast/cont-barna.asp.

\section{Gestión informatizada}

La gestión del centro fue diseñada considerando un sistema de registro clínico electrónico que lograra el objetivo de contar con centros "sin papel". El programa informático adquirido fue el OMI-AP® diseñado específicamente para la APS y que fue adaptado en la PUC para incorporar los elementos del enfoque familiar y también los parámetros de registro estadístico requeridos por el Minsal. El sistema permitió 
conocer antecedentes familiares de los usuarios, realizar control de calidad clínico, control de medicamentos y gestión financiera. El sistema ha permitido, además, desarrollar un panel de gestión asistencial que controle mensualmente indicadores críticos como latencias de atención, tiempos de espera, inasistencias de usuarios y derivaciones. La Tabla $\mathrm{N}^{\circ} 2$ muestra parte de las variables incorporadas en este panel de indicadores.

\section{Efectividad clínica}

Este aspecto involucró un conjunto de dimensiones asociadas con la formación de recursos humanos, disponibilidad de recursos técnicos y trabajo en red. Uno de los aspectos innovadores en esta área fue la incorporación de estrategias de diagnóstico dirigidas a reducir las derivaciones inapropiadas y a mejorar la capacidad resolutiva de los centros. Los criterios definidos para la incorporación de estas innovaciones fueron: relevancia epidemiológica, capacidad de instalación técnica en

TABLA N ${ }^{\circ}$ :

PANEL DE INDICADORES CRÍTICOS DE GESTIÓN CLÍNICA Y ADMINISTRATIVA ANCORA UC

\begin{tabular}{|c|c|c|c|c|c|}
\hline \multicolumn{2}{|r|}{ Perspectiva interna } & \multicolumn{4}{|c|}{ Diciembre 2011} \\
\hline Indicador & Detalle & MTC & JPII & SAH & TOT \\
\hline \multirow[t]{2}{*}{$\begin{array}{l}\mathrm{N}^{\circ} \mathrm{de} \\
\text { inscritos }\end{array}$} & $\begin{array}{c}\mathrm{N}^{\circ} \text { de inscritos activos e } \\
\text { inactivos }\end{array}$ & $\uparrow 23.516$ & $\uparrow 25.351$ & $\uparrow 26.946$ & $\uparrow 75.813$ \\
\hline & $\begin{array}{l}\text { Tasa de consulta médica/ } \\
\text { inscritos validados }\end{array}$ & $\rightarrow 1,58$ & $\downarrow 1,35$ & $\downarrow 1,24$ & $\downarrow 1,39$ \\
\hline Ocupación & $\begin{array}{l}\text { Cupos ocupados/ } \\
\text { cupos disponibles }\end{array}$ & $\rightarrow 0,98$ & $\rightarrow 0,97$ & $\rightarrow 0,98$ & $\rightarrow 0,98$ \\
\hline $\begin{array}{l}\text { Inasisten- } \\
\text { cia }\end{array}$ & Inasistencia/total atenciones & $\uparrow 0,17$ & $\rightarrow 0,16$ & $\rightarrow 0,15$ & $\rightarrow 0,16$ \\
\hline \multirow[t]{2}{*}{$\begin{array}{l}\text { Tiempo de } \\
\text { espera }\end{array}$} & $\begin{array}{l}\text { Promedio de espera en } \\
\text { minutos }\end{array}$ & $\uparrow 15: 49$ & $\uparrow 15: 45$ & $\uparrow 15: 27$ & $\uparrow 15: 40$ \\
\hline & $\begin{array}{l}\text { Atenciones antes de los } 20 \\
\text { min./Todas las atenciones }\end{array}$ & $\uparrow 0,69$ & $\uparrow 0,7$ & $\uparrow 0,70$ & $\uparrow 0,70$ \\
\hline
\end{tabular}

MTC: Centro Ancora Madre Teresa (Puente Alto).

JPH: Centro Ancora Juan Pablo II (La Pintana).

SAH: Centro Ancora San Alberto Hurtado (Puente Alto). 
APS y formación de recursos humanos de APS para operar las innovaciones técnicas.

Las principales innovaciones introducidas fueron: ecografía abdominal, endoscopia digestiva alta, rectosigmoidoscopia y cirugía menor. La ecografía abdominal se incorporó teniendo en cuenta que la patología vesicular es la que genera mayor hospitalización quirúrgica en el país, y muchas veces va asociada a complicaciones (colecistitis, cáncer de vesícula) que son prevenibles al contar con un diagnóstico ecográfico precoz (Puschel et al., 2002). La endoscopia digestiva alta se incorporó debido a que las patologías gastrointestinales, tales como úlcera gastroduodenal y cáncer gástrico, representan uno de los mayores problemas de salud del país (Ministerio de Salud, 2012a) y su diagnóstico endoscópico precoz puede prevenir complicaciones y reducir la mortalidad por estas causas (Miyamoto et al., 2007; Ang et al., 2010). La patología anorrectal y la cirugía menor han sido tradicionalmente áreas de carencia en el sistema de salud y que generan alta demanda de atención. Existe evidencia que muestra el beneficio de abordar estas patologías en APS (Serra et al., 2010).

\section{Formación}

El desarrollo de capacidad formativa fue un aspecto relevante del proyecto dados la importancia de contar con recursos humanos capacitados específicamente en APS, la insuficiencia de ellos en Chile y el predominio de formación hospitalaria de los profesionales en salud (Baas, 2011). La APS no ha sido tradicionalmente un área relevante de formación de pre o postgrado en carreras médicas o del área psicosocial $\mathrm{y}$ tiene muchas dificultades para atraer profesionales que se desarrollen en ella. La alta carga asistencial, debido al elevado volumen de atención en APS, es una barrera significativa para desarrollar actividades docentes. El desafío del proyecto fue el de combinar una actividad asistencial eficiente y efectiva con formación profesional de alta calidad en APS que aportara al desarrollo de Ancora.

Para estimular la formación en APS la PUC creó, con fondos de la donación y propios, un programa de becas de especialización en salud familiar para profesionales en medicina, enfermería, psicología y trabajo social. Estos profesionales podían optar al financiamiento de programas de residencias o magíster vinculados a salud familiar. 
Al finalizar su programa debían estar disponibles para ser contratados por un período equivalente al de la duración de su programa en caso de ser solicitado por los centros. La actividad docente también incluyó estudiantes de pregrado de medicina y enfermería en APS. El desarrollo docente obligó a diseñar espacios físicos especiales en los centros que incluyeron salas de supervisión con espejo, salas de docencia tutorial y soporte informático (Tabla $\mathrm{N}^{\circ} 1$ ). Desde el punto de vista operativo, la actividad docente implicó crear agendas de atención separadas para la docencia y asistencia, resguardar espacios y tiempos de discusión docente y establecer sistemas de financiamiento docentes y asistenciales diferenciados.

\section{Desafios políticos de implementación}

Desde el punto de vista político, el proyecto Ancora implicaba el desarrollo de un sistema mixto de salud en la APS a través de la colaboración entre una institución académica sin fines de lucro, como la PUC, y el sistema público de salud. Esta experiencia era muy limitada en Chile e implicaba un precedente en políticas públicas que produjo reacciones, muchas veces contradictorias. El proyecto originó una intensa resistencia inicial de los gremios de trabajadores de la APS agrupados en la Confusam, que presionaron fuertemente a las autoridades ministeriales por el cierre de los centros o por la limitación de su desarrollo futuro. La Confusam veía en el proyecto un impulso privatizador que podía afectar legítimos intereses gremiales y afectar el acceso y calidad de la atención de salud de la población (diario La Nación, 2007). Por otra parte, el proyecto contaba con el apoyo de las autoridades ministeriales de la época y con un importante reconocimiento internacional (Chu et al., 2006).

Las consecuencias de la tensión política generada fueron de diverso orden. Por una parte, el proyecto Ancora se limitó a tres centros en contraste con los seis originalmente contemplados. Por otra parte, la interacción de los gestores del proyecto con las asociaciones gremiales y la Asociación Chilena de Municipalidades generó un diálogo productivo que derivó en el desarrollo de un trabajo más colaborativo. Una de las expresiones de este trabajo fue el planteamiento de una propuesta conjunta de financiamiento para la APS en la cual académicos de la PUC aportaron con uno de los dos estudios que sirvieron de base para la negociación con el Minsal (Frente de Defensa de la Salud, 2010). 


\section{Metodología de evaluación}

La evaluación del impacto de los centros Ancora UC incluyó tres dimensiones: satisfacción usuaria, efectividad clínica e impacto económico. La evaluación se realizó mediante tres aproximaciones metodológicas.

\section{Satisfacción usuaria}

La satisfacción usuaria de la población de los centros Ancora UC se evaluó en el contexto del proyecto FONIS SA07I20032 ("Evaluación del modelo de salud familiar en Atención Primaria desde la perspectiva de los usuarios", Peñaloza et al., 2008). En este proyecto se realizó un estudio de corte transversal, observacional y concurrente con el objetivo de evaluar la percepción de los usuarios del modelo de salud familiar en una muestra nacional de centros de atención primaria. Para realizar la evaluación se aplicó el instrumento validado "Encuesta de expectativas, percepción y satisfacción usuaria con el modelo de salud familiar". Este instrumento mide calidad percibida ("experiencia"), expectativas con los servicios de salud ("expectativas") y satisfacción lograda ("aceptabilidad") de los principales componentes del modelo de salud familiar. Los parámetros de evaluación de este modelo fueron acordados con el Minsal e incluyen: accesibilidad, continuidad del cuidado, resolutividad, enfoque preventivo, enfoque promocional, participación comunitaria y enfoque psicosocial.

El estudio contempló un muestreo bietápico complejo, obteniéndose una muestra representativa de usuarios a nivel nacional agrupados en cuatro estratos: Cesfam (Centro de Salud Familiar), centros no-Cesfam, centros rurales y centros urbanos. De un universo de 508 centros a nivel nacional (355 urbanos y 153 rurales) se seleccionaron 39 centros (27 urbanos y 12 rurales). Éstos incluyeron 18 Cesfam y 21 no-Cesfam. En cada centro se seleccionaron individuos consultantes por muestreo aleatorio simple mediante cuotas preestablecidas por sexo y rango etario. El estudio incluyó una muestra aleatoria de un total de 1.544 usuarios de los 39 centros, estratificados por sexo y edad. Cada encuestado evaluó los centros usando una escala entre 1 y 7 puntos. El tamaño muestral de este estudio se definió considerando una probabilidad de error tipo alfa de 0,05 y una probabilidad de error tipo beta de 0,2. El 
estudio fue diseñado con un poder de 0,8 para detectar diferencias de al menos 5\% (0,35 puntos) en la satisfacción global de atención de los usuarios.

\section{Efectividad clínica}

La efectividad clínica se analizó desde un estudio externo encargado a la Consultora Epidemiología y Gestión Ltda. que exploró el acceso y la resolutividad clínica de los centros durante el año 2010 (Infante y Salas, 2011). En este estudio se utilizó un diseño transversal que tuvo como objetivo comparar la resolutividad de los centros Ancora UC de las comunas de Puente Alto y La Pintana con la de los centros municipales ubicados en las mismas comunas del área suroriente de Santiago. La resolutividad clínica se definió como la capacidad de los centros de solucionar los problemas de atención médica a nivel primario y, por tanto, evitar la derivación de sus consultas a las especialidades del nivel secundario, servicios de urgencia y hospital. La resolutividad ha sido considerada como un indicador esencial de efectividad clínica (SicrasMainar et al, 2007) y ha sido identificada como un aspecto deficiente en la APS chilena (Roman, 2011).

En la evaluación de la efectividad clínica se utilizaron dos aproximaciones metodológicas complementarias: una epidemiológica y la otra georreferencial. En la aproximación epidemiológica se analizaron datos secundarios provenientes del Departamento de Estadísticas e Información de Salud (DEIS) del Ministerio de Salud. Además, se obtuvieron datos provenientes de los registros estadísticos del SSMSO, hospitales Sótero del Río y Padre Hurtado. Por otra parte, se revisó la información de registros estadísticos comunales de Puente Alto y La Pintana. Por último se revisaron las estadísticas de los servicios de atención primaria de urgencia (SAPU-SUC) de Puente Alto y La Pintana.

La información requerida apuntó a obtener el número de atenciones de especialidad ambulatoria, hospitalizaciones y consultas en los servicios de urgencia hospitalario y municipal provenientes de usuarios registrados en los centros Ancora y municipales. La información entregada fue contrastada con el domicilio de los usuarios y su número de RUT, lo que permitió confirmar el centro en el cual se encontraban registrados. Esto hizo posible además realizar un análisis georreferencial que graficara los flujos de consulta desde y hacia los centros Ancora UC 
y municipales en las comunas estudiadas. En cada fuente explorada se intentó obtener el máximo de información disponible respecto al origen de las atenciones. Dadas las limitaciones existentes en la disponibilidad de información, se buscó utilizar varias fuentes para contrastar los resultados obtenidos. De esta forma, se combinaron en el resultado final los datos secundarios (DEIS) con los basados en el registro de atenciones del hospital y los sociodemográficos (RUT y direcciones) para definir el origen (Ancora UC o municipal) de las atenciones realizadas. La metodología utilizada no consideró entrevista a pacientes o análisis de información de fichas clínicas. Sólo se analizaron listados de información desde las bases existentes en los diferentes centros mencionados.

\section{Impacto económico}

El impacto económico del proyecto fue estudiado mediante la comparación entre Ancora UC y centros municipales de tres parámetros: montos de ingreso per cápita, gastos devengados en el nivel primario y gastos asociados a derivación. Estos últimos incluyeron los gastos generados por prestaciones realizadas a usuarios de Ancora UC y centros municipales en el nivel secundario de especialidad, servicio de urgencia y hospital. De esta forma, el balance económico se realizó desde una perspectiva del sistema de salud evaluando el impacto de la intervención Ancora UC sobre el total de la red a determinados niveles de ingreso asociados al aporte per cápita. No se consideraron en este análisis los costos indirectos como, por ejemplo, los representados por el valor del tiempo destinado a acudir a urgencia, consulta de especialidad o los asociados a ausencia laboral por hospitalizaciones.

Las fuentes de financiamiento utilizadas para el análisis del año 2010 fueron el convenio establecido entre el SSMSO y Ancora UC en marzo de 2010 y los datos de ingresos totales del área salud. Se descontaron en el análisis las transferencias del municipio al sector, reportadas en el Sistema Nacional de Información Municipal (Sinim, 2010) para el año 2010. Se consideró específicamente la información disponible para las comunas de La Pintana (código 13112) y Puente Alto (código 13201). En la misma fuente se estimó el gasto total en salud devengado para el año 2010 y la población inscrita validada en las dos comunas estudiadas. Esta información se comparó con los gastos registrados en el balance económico de Ancora UC y presentados a la gerencia de 
finanzas de la Facultad de Medicina (PUC) para el año 2010. Esto permitió realizar una comparación del gasto per cápita en salud municipal de cada comuna con el gasto per cápita para la red Ancora UC basado en la información de población inscrita validada por Fonasa para el año 2009. A esta información se agregó el gasto estimado en salud generado por las derivaciones extras (margen de derivación) de los centros primarios a los niveles secundario, urgencia y hospitalario en el año 2010. El costo de las prestaciones en el nivel secundario y consultas de urgencia hospitalaria se estimó en $\$ 15.000$, el de urgencia SAPU en $\$ 7.500$ y el costo por día/cama hospital se estimó en $\$ 60.860$ sobre la base de los aranceles de modalidad de atención institucional (MAI) de Fonasa (2011).

\section{Resultados}

\section{Perfil de la población}

La Tabla $\mathrm{N}^{\circ} 3$ presenta el perfil basal de la población inscrita en Ancora UC y el de la población municipal en el año 2010. Puede observarse que tanto a nivel municipal como en Ancora UC existe un predominio de población inscrita femenina. Al comparar las edades, la tabla muestra que la población inscrita en Ancora UC es, en promedio, menor que la inscrita en los centros municipales. Esta diferencia es estadísticamente significativa $(\mathrm{p}<0,001)$. Las mayores diferencias se observan en el grupo de lactantes (0-2 años) y adultos mayores (65-79 años) en la comuna de La Pintana. En relación a nivel socioeconómico, el indicador de ingreso utilizado corresponde al nivel previsional de Fonasa. La población perteneciente a Fonasa "A" corresponde a la categoría de menores ingresos y está constituida por población que carece de ingresos estables o de un trabajo formal. El porcentaje de la población perteneciente a la categoría de menor ingreso es similar al comparar los centros Ancora UC de Puente Alto y los municipales (30,9\% vs. 30,8\%). Sin embargo, en La Pintana, el porcentaje de población de menor ingreso inscrita en Ancora UC (60\%) es mayor que el comunal (46,7\%). Esta diferencia es estadísticamente significativa $(\mathrm{p}<0,001)$. Al comparar ambas comunas en forma global, puede observarse que el porcentaje de población de menores ingresos en La Pintana es mayor que el de Puente Alto. El centro Ancora UC de La Pintana es el que agrupa la mayor po- 
TABLA N ${ }^{\circ}$ 3: $\quad$ PERFIL BASAL DE LA POBLACIÓN ANCORA UC Y COMUNAL EN EL AÑO 2010

\begin{tabular}{lccccc}
\hline \multicolumn{1}{c}{ Variable } & $\begin{array}{c}\text { Comuna } \\
\text { Puente Alto }\end{array}$ & $\begin{array}{c}\text { Ancora } \\
\text { Puente Alto }\end{array}$ & $\begin{array}{c}\text { Comuna } \\
\text { La Pintana }\end{array}$ & $\begin{array}{c}\text { Ancora } \\
\text { La Pintana }\end{array}$ & $\begin{array}{c}\text { Diferencia } \\
\text { estadística }\end{array}$ \\
\hline Total población inscrita & 379.080 & 46.535 & 163.833 & 21.307 & \\
$\begin{array}{l}\text { Género: \% población } \\
\text { femenina }\end{array}$ & 54,0 & 53,9 & 53,9 & 51,5 & p: NS \\
Edad: promedio (DE) & $32,4(20,8)$ & $30,9(20,6)$ & $31,7(20,8)$ & $28,8(19,2)$ & $\mathrm{p}<0,001$ \\
0-2 años (\%) & 2,9 & 3,5 & 2,3 & 4,4 & \\
3-10 años (\%) & 12,6 & 13,8 & 13,9 & 13,8 & \\
11-20 años (\%) & 18,4 & 18,4 & 19,5 & 19,58 & \\
21-64 años (\%) & 58,7 & 56.8 & 56,7 & 58,3 & \\
65-79 años (\%) & 6,1 & 5,6 & 6,5 & 3,3 & \\
80 y + años (\%) & 1,3 & 1,3 & 1,0 & 0,7 & \\
Nivel SE* & & & & & P $<0,001$ \\
Fonasa A (\%) & 30,9 & 30,8 & 46,7 & 60,0 & \\
(Menor ingreso) & & & & & \\
Fonasa B (\%) & 27,8 & 26,2 & 23,7 & 19,0 & \\
Fonasa C (\%) & 20,6 & 23,4 & 15,0 & 12,8 & \\
Fonasa D (\%) & 20,7 & 19,3 & 14,6 & 8,3 & \\
(Mayor ingreso) & & & & & \\
\hline
\end{tabular}

* Nivel SE: nivel socioeconómico. Se clasificó de acuerdo a nivel de Fonasa.

Fonasa A: personas que carecen de ingresos o de un trabajo formal. Tienen acceso y gratuidad a las atenciones de salud en la red pública.

Fonasa B: personas que reciben un ingreso menor que el sueldo mínimo. Tienen acceso y gratuidad a las atenciones de salud en la red pública.

Fonasa $\mathrm{C}$ y D: personas que reciben un ingreso mayor que el sueldo mínimo. Pueden utilizar sistema de libre elección con copagos establecidos.

blación de menores ingresos de la muestra. En él, más de la mitad de la población se encuentra en el grupo de mayor vulnerabilidad social.

\section{Satisfacción usuaria}

En el estudio nacional que evaluó la satisfacción de los usuarios de centros de salud, los tres centros Ancora recibieron un puntaje promedio de 6,13. Este puntaje es significativamente mejor que el promedio total de la muestra nacional, que fue de 5,2. Al considerar el porcentaje de pacientes que evaluaron los centros con puntaje igual o mayor que 6, los tres centros Ancora ocuparon el primer lugar (90\%), quinto lugar $(75 \%)$ y sexto $(68 \%)$ del total de 41 centros evaluados. 
Esta mejor evaluación en el puntaje fue también concordante con la evaluación en las áreas específicas del modelo. En todos los componentes evaluados, los centros Ancora fueron evaluados significativamente mejor que el promedio de la muestra utilizada.

\section{Efectividad clínica}

Los resultados de efectividad clínica se presentan en la Tabla $\mathrm{N}^{\circ}$ 4. Aquí se muestran las tasas absolutas de consulta en urgencia y derivación de los centros comunales y Ancora UC el año 2010. En ella destaca que los centros Ancora UC obtuvieron una mayor efectividad clínica, estimada por los indicadores de resolutividad basados en consulta en urgencia comunal (SAPU), urgencia hospitalaria, derivación a especialidades y atención en el nivel secundario. El mayor efecto se produjo en la reducción relativa de consultas en urgencia SAPU, que osciló en rangos entre $78 \%$ y $62 \%$ en Puente Alto y La Pintana respectivamente. La consulta en urgencia hospitalaria fue entre $19 \%$ y $25 \%$ menor en los centros Ancora UC respecto de los centros municipales. Las diferencias observadas se mantuvieron al ajustar por edad y nivel socioeconómico en el análisis univariado y multivariado $(\mathrm{p}<0,001)$. Esto significó que Ancora UC ahorró, en promedio, un total de 6.394 consultas de urgencia a la red hospitalaria pública de salud durante el año 2010.

La derivación y atención de la población Ancora UC a especialidades de nivel secundario también fue significativamente menor respecto a la población inscrita en centros municipales. Este efecto se tradujo en una menor generación de lista de espera en especialidades, que en 2010 significó, en promedio, un ahorro de 2.477 derivaciones. La reducción en atención en especialidades también fue significativa, siendo en promedio 13\% menor en los Ancora UC de Puente Alto y 41\% menor en La Pintana. Las diferencias observadas fueron estadísticamente significativas y se mantuvieron al ajustar por las diferencias basales de edad y nivel socioeconómico, tanto en el análisis univariado como en el multivariado ( $\mathrm{p}<0,001)$. La Tabla $\mathrm{N}^{\circ} 4$ muestra los intervalos de confianza de los estimadores obtenidos ajustados por edad y nivel socioeconómico.

En relación a egresos hospitalarios, el efecto promedio observado siguió la misma tendencia que el observado en consultas de urgencia 


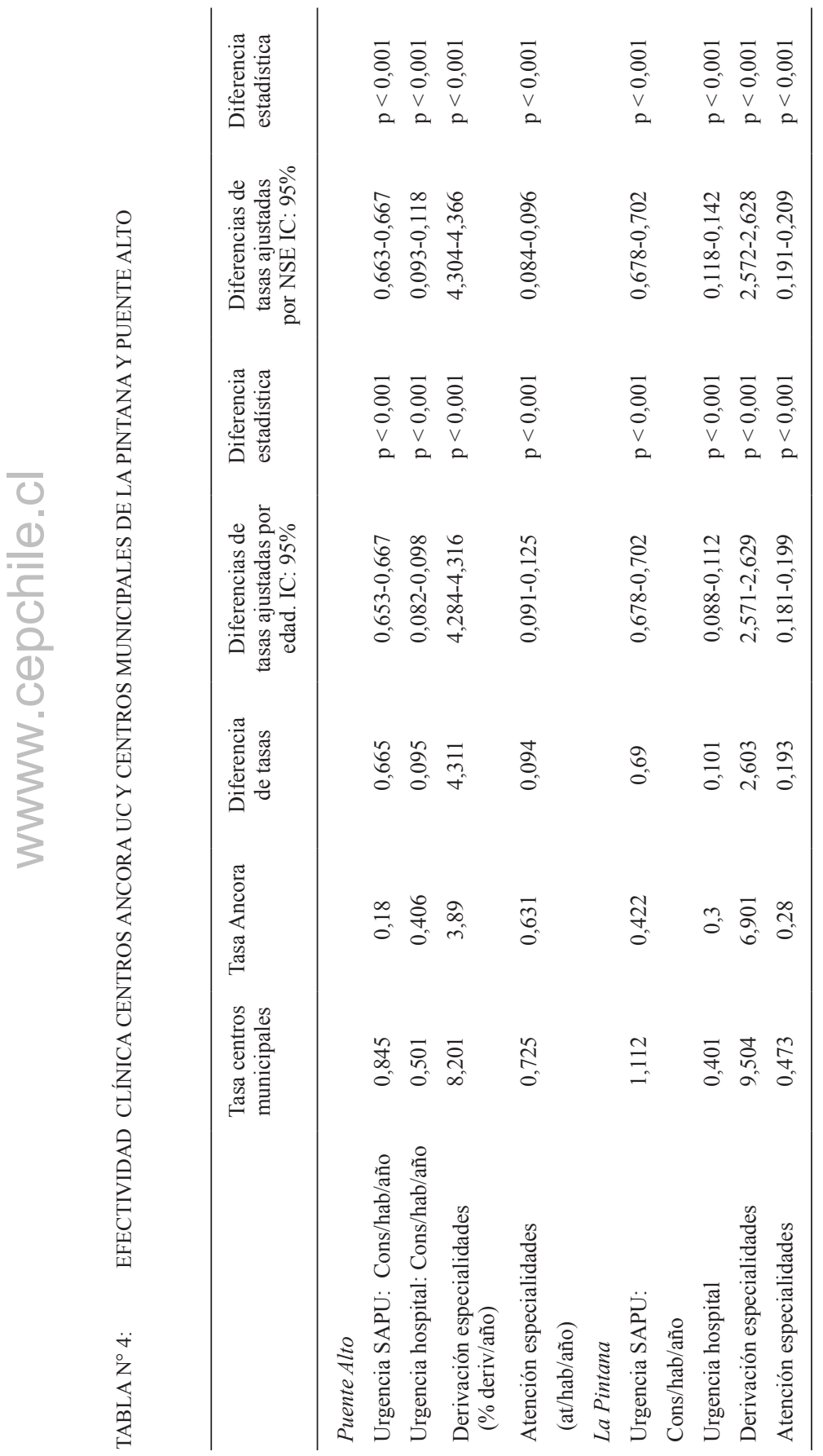


y derivaciones a nivel secundario. Los centros Ancora generaron 30\% menos de hospitalizaciones que los centros municipales. Este porcentaje es consistente con la reducción observada en consultas de urgencia. Esta reducción representó un ahorro de 9.500 días cama durante el año 2010.

\section{Impacto económico}

Los resultados respecto a impacto económico pueden observarse en las Tablas $\mathrm{N}^{o s} 5$ y 6 . La Tabla $\mathrm{N}^{\circ} 5$ muestra los ingresos y gastos totales reportados por el Sinim y convenio SSMSO-PUC para el año 2010. Además, se presenta el gasto per cápita basado en la población inscrita en las comunas de La Pintana, Puente Alto, La Florida, San Joaquín y Ancora UC validada por Fonasa el año 2009. Puede observarse que, comparativamente, Ancora UC recibió un ingreso per cápita inferior y generó un gasto menor que el de las comunas de comparación durante el año 2010.

La Tabla $\mathrm{N}^{\circ} 6$ presenta el ahorro asociado a las diferencias en efectividad clínica y menor ingreso per cápita de los centros Ancora UC respecto de los centros municipales. Puede observarse que Ancora UC generó ahorros asociados a la menor consulta en el nivel secundario, urgencia hospitalaria, SAPU y egresos hospitalarios que ascendieron a 1.017,1 millones de pesos el año 2012. Aproximadamente la mitad de

TABLA N ${ }^{\circ}$ 5: $\quad$ INGRESOS Y GASTOS TOTALES PER CAPITA ANCORA UC Y MUNICIPALES SERVICIO DE SALUD METROPOLITANO SURORIENTE, SANTIAGO, 2010

\begin{tabular}{llccccc}
\hline Código* & Municipio & $\begin{array}{c}\text { Ingresos: Trans- } \\
\text { ferencias desde } \\
\text { salud sin aporte } \\
\text { municipal }\end{array}$ & $\begin{array}{c}\text { Gasto total } \\
\text { en salud }\end{array}$ & $\begin{array}{c}\text { Inscritos } \\
2009\end{array}$ & $\begin{array}{c}\text { Per càpita } \\
\text { mes/ } \\
\text { ingreso }\end{array}$ & $\begin{array}{c}\text { Per càpita } \\
\text { mes/ } \\
\text { gasto }\end{array}$ \\
\hline 13112 & La Pintana & $\$ 11.292 .351 .000$ & $\$ 10.287 .211 .000$ & 191.097 & $\$ 4.924$ & $\$ 4.486$ \\
13201 & Puente Alto & $\$ 16.585 .901 .000$ & $\$ 18.207 .776 .000$ & 410.349 & $\$ 3.368$ & $\$ 3.698$ \\
13110 & La Florida & $\$ 15.016 .405 .000$ & $\$ 16.914 .408 .000$ & 320.220 & $\$ 3.908$ & $\$ 4.402$ \\
13129 & San Joaquin & $\$ 4.406 .359 .000$ & $\$ 5.171 .420 .000$ & 84.959 & $\$ 4.322$ & $\$ 5.072$ \\
& Ancora UC & $\$ 2.531 .648 .000$ & $\$ 2.744 .361 .000$ & 67.329 & $\$ 3.133$ & $\$ 3.397$ \\
\hline
\end{tabular}

* Código Sistema Nacional de Información Municipal (SINIM). 
TABLA N ${ }^{\circ}$ 6: $\quad$ AHORROS ASOCIADOS A EFECTIVIDAD CLÍNICA Y NIVELES DE INGRESO CENTROS ANCORA UC Y MUNICIPALES AÑO 2010

\begin{tabular}{lccccccc}
\hline & $\begin{array}{c}\text { Atención en } \\
\text { especiali- } \\
\text { dades nivel } \\
\text { secundario }\end{array}$ & $\begin{array}{c}\text { Atención } \\
\text { urgencia } \\
\text { hospital }\end{array}$ & $\begin{array}{c}\text { Atención } \\
\text { urgencia } \\
\text { SAPU }\end{array}$ & $\begin{array}{c}\text { Egresos } \\
\text { hospita- } \\
\text { larios }\end{array}$ & $\begin{array}{c}\text { Total } \\
\text { ahorro } \\
\text { por des- } \\
\text { empeño }\end{array}$ & $\begin{array}{c}\text { Menor } \\
\text { transfe- } \\
\text { rencia } \\
\text { per cápita }\end{array}$ & $\begin{array}{c}\text { Total } \\
\text { ahorro } \\
\text { anual }\end{array}$ \\
\hline $\begin{array}{l}\text { Arancel } \\
\text { MAI }\end{array}$ & $\$ 15.000$ & $\$ 15.000$ & $\$ 7.500$ & $\$ 60.860$ & & & \\
$\begin{array}{l}\text { Número } \\
\text { de presta- } \\
\text { ciones } \\
\text { ahorradas } \\
\begin{array}{l}\text { Ahorros } \\
\text { totales } \\
\text { (\$ millones) }\end{array}\end{array}$ & 2.285 & 6.394 & 41.166 & 9.500 & & & \\
\hline
\end{tabular}

MAI: Modalidad de atención institucional.

este ahorro se debió a egresos hospitalarios ( $\$ 578,17$ millones). Si a este ahorro se agrega el asociado al menor ingreso per cápita de Ancora UC versus los centros municipales, el total de ahorro se eleva a $\$ 1.697,1 \mathrm{mi}-$ llones anuales.

\section{Discusión}

Este estudio muestra que un modelo de atención primaria basado en los principios de enfoque familiar, efectividad clínica y capacidad formativa es implementable en Chile y produce beneficios en términos de satisfacción usuaria, resolutividad e impacto económico. El estudio es relevante dado que incorpora un concepto de APS innovador, que se aleja de la definición tradicional de atención básica introduciendo variables de interdisciplinariedad, tecnología y formación de recursos humanos. Éstas son las variables que, en diversas experiencias internacionales, se han asociado con una APS "fuerte", capaz de producir un efecto positivo en la red de salud (Pasarín et al., 2007; Starfield, 2012).

La necesidad de desarrollar sistemas de salud articulados desde una atención primaria "fuerte" ha sido enfatizada por la Organización Mundial de la Salud (2008) por dos razones esenciales. En primer lugar, por el cambio del perfil epidemiológico de la población caracterizado por el predominio de enfermedades crónicas que requieren sistemas integrales de atención. En segundo lugar, por la evidencia existente 
que muestra que sistemas basados en atención hospitalaria y de subespecialidad son menos efectivos y generan mayores gastos que los basados en APS (Starfield et al., 2005). Experiencias como las de Indonesia o Austria muestran las consecuencias negativas de modelos de salud basados en atención hospitalaria y con una APS “débil" (Simms y Rowson, 2003; Stigler et al., 2012). Chile está experimentando cambios epidemiológicos similares a los de países con alto desarrollo socioeconómico (Ministerio de Salud, 2011). Existe acuerdo en las autoridades ministeriales, legislativas y académicas sobre el insuficiente desarrollo del sistema de salud en atención primaria y la necesidad de fortalecer el modelo en esta área (Cámara de Diputados de Chile, 2012).

Los resultados del estudio pueden contribuir a la discusión sobre las estrategias a seguir para fortalecer el nivel primario. Las significativas reducciones observadas en derivaciones a nivel secundario (27\%-55\%), consultas de urgencia hospitalaria (19\%-25\%), urgencia SAPU (62\%-70\%) y egresos hospitalarios (30\%) son concordantes con las observadas a nivel internacional. Probst et al. (2009) observaron una reducción de $16 \%$ en hospitalizaciones en municipalidades con alto desarrollo de APS vs. aquellas con bajo desarrollo en ocho estados en EE.UU. Guttman et al. (2010) encontraron 59\% de reducción en consultas de urgencia y hospitalización en niños que habitaban áreas con APS más desarrollada en Canadá. En la misma línea, Rosenberg et al. (2012) observaron 33\% de reducción acumulada de ingresos y reingresos hospitalarios durante el período 2008-2010 al comparar un nuevo modelo integral de APS vs. el modelo tradicional. Finalmente, Rosano et al. (2012) encontraron una relación lineal y significativa entre mayor desarrollo de APS y hospitalizaciones evitadas en una revisión sistemática que incluyó 51 estudios. Resultados similares se han observado en estudios que han evaluado modelos integrales de APS en países de ingresos medios o bajos (Macinko et al., 2009). En Brasil, la evaluación del proyecto "Programa de Salud Familiar" realizado entre los años 1999 y 2007 demostró que los centros incluidos en él obtenían una reducción significativa de $20 \%$ en hospitalizaciones asociadas a enfermedades crónicas tales como enfermedad coronaria, cerebrovascular y asma, comparados con centros de atención habitual (Macinko et al., 2010; Dourado et al., 2011).

Uno de los factores que parece relevante para explicar los beneficios de una APS "fuerte" es el tipo de profesional médico que trabaja en 
estos centros. En una muestra nacional en EE. UU., Philips et al. (2009) demostraron que el médico familiar generaba $50 \%$ menos de gasto en hospitalizaciones y visitas a servicios de urgencia y $30 \%$ menos de gasto en medicamentos que un médico internista o pediatra general trabajando en APS. Otros estudios también apuntan a los beneficios del médico familiar como el más apropiado para trabajar en APS (Shi et al., 2003; Bertakis, 1999). La experiencia de Ancora, donde el médico familiar es el actor predominante de la atención médica, es concordante con los resultados descritos. Las políticas ministeriales chilenas actuales de formación de recursos médicos parecen ir en la línea contraria a la evidencia mencionada. Ellas privilegian la formación de médicos con perfil hospitalario para APS y no ofrecen actualmente posibilidades de formación directa para recién egresados en medicina familiar. La falta de incentivos en esta área agrava la carencia de estos profesionales en APS y mantiene un sistema de formación superado por los países con mejores indicadores de salud en el mundo.

En relación al impacto económico del proyecto, los resultados muestran que los centros Ancora generan ahorros significativos al sistema, especialmente en atenciones de nivel secundario, hospitalizaciones y urgencia. Estos ahorros "deseados" de una APS más desarrollada se han observado también en otras experiencias internacionales (Starfield, 2012). Sin embargo, los resultados de este estudio también muestran que se producen ahorros "no deseados" y que tienen que ver con el (des)financiamiento del proyecto. La pérdida progresiva de ingresos del proyecto por menor reajuste per cápita ha implicado restricción en el desarrollo de innovaciones que pueden incrementar el efecto positivo observado y que amenazan la viabilidad del proyecto. La experiencia internacional muestra que una reducción del financiamiento de la APS se traduce en un empeoramiento del estado de salud de la población y un deterioro de la razón costoefectividad del sistema (Simms y Rowson, 2003; Starfield, 2005).

La experiencia desarrollada en el proyecto Ancora UC presentó diversas dificultades de implementación que son importantes de considerar al analizar su potencial replicabilidad. A la resistencia política inicial descrita debe sumarse un grado inicial de sospecha de la población respecto a los intereses económicos de una entidad externa como la PUC en implementar un sistema de atención de salud. Esto se vio reflejado en un lento proceso de inscripción por parte de la población 
y requirió un intenso trabajo comunitario por parte de los equipos de salud. Otra dificultad importante consistió en la integración de un sistema externo como Ancora UC a una red pública y municipal de larga trayectoria. La desconfianza inicial de la red hacia Ancora UC pudo ser superada por un explícito apoyo de las autoridades municipales de La Pintana, Puente Alto y del SSMSO que sugirieron estrategias y ayudaron a integrar los centros Ancora UC en la red pública de salud manteniendo su identidad.

\section{Limitaciones del estudio}

Este estudio tiene limitaciones metodológicas que son importantes de considerar. En primer lugar, se trata de un estudio de diseño transversal y por tanto puede no reflejar la variabilidad de resultados a lo largo del tiempo. En segundo lugar, el muestreo de los centros comparadores para evaluar la efectividad clínica fue por conveniencia, no aleatorio. Las poblaciones beneficiarias de los centros de comparación podrían tener diferencias no expresadas en la evaluación y que impacten en los resultados. Sin embargo, la comparación de los centros Ancora UC con los centros municipales de las propias comunas pareció la más apropiada para asegurar que ambas poblaciones estuvieran sometidas a los mismos sistemas de referencia secundaria, urgencia y hospitalaria. Las mayores diferencias poblacionales basales observadas al comparar efectividad clínica entre centros Ancora y municipales se observaron en la comuna de La Pintana. En ella, la población Ancora inscrita era significativamente más pobre y de menor edad promedio respecto a la de los centros municipales. Sin embargo, al ajustar por edad y nivel socioeconómico, no se observaron diferencias en los resultados asociados a derivaciones a especialidades, consulta en urgencia municipal o consulta en urgencia hospitalaria.

La información disponible basada en datos secundarios no permitió realizar otros ajustes posibles tales como perfil basal de problemas de salud o análisis de resultados más específicos de egresos hospitalarios según condiciones seleccionadas. De esta forma, si por ejemplo alguno de los centros municipales tuviera una alta y desproporcionada tasa de accidentes como causa de hospitalización, el beneficio observado de Ancora UC aparecería sobrevalorado. La comparación de la población de los centros Ancora UC con la de los centros de las propias comunas 
donde éstos están ubicados puede reducir esta posibilidad. Más aún, el centro de mayor vulnerabilidad social de la muestra es precisamente el Ancora UC ubicado en El Castillo, La Pintana, donde las condiciones sociales son más adversas que en los centros de comparación.

\section{Conclusión}

El modelo de salud Ancora UC basado en los principios de enfoque familiar, gestión informatizada, efectividad clínica y formación profesional puede generar beneficios a la red local de salud. El modelo se asocia a altos niveles de satisfacción usuaria, mayor efectividad clínica y ahorros derivados de una menor demanda de atención de urgencias, especialidades y hospitalización. Los resultados obtenidos van en la misma dirección que los observados en países de alto desarrollo en salud como Canadá o Inglaterra y países emergentes con proyectos similares como Brasil. Una política pública eficiente y efectiva en salud en Chile debiera considerar la modificación del modelo actual de APS y situarla como eje articulador de la red de salud.

\section{REFERENCIAS}

Ang, T. L., C. J. Khor y T. Gotoda (2010). "Diagnosis and Endoscopic Resection of Early Gastric Cancer”. Singapore Medical Journal 51, 2: 93-100.

Asociación Chilena de Municipalidades (2009). "Estudio Diagnóstico de la Situación de Gestión de Salud en las Entidades Administradoras Municipales e Identificación de Buenas Prácticas Locales". Resumen ejecutivo. Santiago, abril 2009. http://www.munitel.cl/file_admin/ archivos_munitel/salud/salud38.pdf.

Baas, C. (2011) "Desarrollo de Recursos Humanos en Atención Primaria de Salud". Seminario de Salud Municipal. Asociación Chilena de Municipalidades, Colegio Médico de Chile. Santiago 20 de abril de 2011. http://www.munitel.cl/eventos/seminarios/html/documentos/2011/ seminario_de_salud_municipal_macro_zona_centro_quillota/PPT13.pdf. - (2012) "Modelo de Salud Familiar en Chile y Mayor Resolutividad de la Atención Primaria de Salud: ¿Contradictorios o Complementarios?" Medwave 12,11; e5571 doi: 10.5867/medwave.2012.11.5571.

Bertakis, K, L. J. Helms, R. Azari, E. Callahan y J. A. Robbins (1999). 'Differences between Family Physicians' and General Internists Medical Charges". Medical Care 37, 1: 78-82.

Cámara de Diputados de Chile, Ministerio de Salud, Escuela de Salud Púbica Universidad de Chile (2012). Seminario: "Los problemas de Salud de Chile para el 2040”. Cámara de Diputados de Chile. 31 de Julio de 2012. 
http:/www.bcn.cl/ciencia-e-innovacion/los-problemas-de-salud-de-chilepara-el-2040.

Chu, M., M. Koljatic y M. Silva (2006). "Ancora: A Private University Providing Healthcare for the Poor". Harvard Business School. Case Number 306-088.

DEIS (Departamento de Estadísticas e Información de Salud) (2009). "Estadísticas de Atenciones y Recursos para la salud Chile, años 2005 a 2009".

Doherty, W. J. y M. A. Baird (1983). Family Therapy and Family Medicine towards the Primary Care of Families. New York: Guildford Press.

Dourado, I., V. B. Oliveira, R. Aquino, P. Bonolo, M. F. Lima-Costa, M. G. Medina, E. Mota, M. A. Turci y J. Macinko (2011). "Trends in Primary Health Care-Sensitive Conditions in Brazil: The Role of the Family Health Program (Project ICSAP-Brazil)". Medical Care 49, 6: 577-584.

FONASA (2011). "Modalidad de Atención Institucional, 2011". http://www. fonasa.cl/wps/wcm/connect/internet/sageneral/prestadores/aranceles/ aranceles.

Frente de Defensa de la Salud (2010). "Financiamiento de la Atención Primaria de Salud". http://frentedefensasalud.bligoo.cl/content/view/1043778/ Informe-acerca-del-Financiamiento-de-la-Atencion-Primaria-de-Salud.

Guttmann, A., S. A. Shipman y K. Lam (2010). "Primary Care Physician Supply and Children's Health Care Use, Access, and Outcomes: Findings from Canada". Pediatrics 125: 1.119-1.126.

Hidalgo, C. G. y E. Carrasco (2000). Salud Familiar: Un Modelo de Atención Integral en la Atención Primaria. Ediciones de la Universidad Católica de Chile, 2000.

Infante, A. y X. Salas (2011). "Evaluación de desempeño de consultorios Ancora: Equidad de acceso y resolutividad". Documento de Trabajo, Consultora Epidemiología y Gestión.

Kringos, D. S., W. G, Boerma, A. Hutchinson, J. Van der Zee. y P. Groenewegen (2010). "The Breadth of Primary Care: A Systematic Literature Review of its Core Dimensions”. Biomedcentral Health Services Research 10: 65-78.

La Nación. "Confusam Anunció Paro Nacional por Privatización de la Salud Pública”, domingo 17 de junio 2007, diario La Nación, Santiago de Chile.

Lin, E., M. Von Korff, P. Ciechanowski, D. Peterson, E. J. Ludman, C. M. Rutter, M. Oliver, B. A. Young, J. Gensichen, M. McGregor, D. K. McCulloch, E. H. Wagner y W. J. Katon (2012). "Treatment Adjustment and Medication Adherence for Complex Patients with Diabetes, Heart Disease, and Depression: A Randomized Controlled Trial". Annals of Family Medicine 10, 1: 6-14.

Macinko, J., B. Starfield y T. Erinosho (2009). "The Impact of Primary Healthcare on Population Health in Low - and Middle- Income Countries". Journal Ambulatory Care Management 32, 2: 150-171.

Macinko, J., I. Dourado, R. Aquino, P. de F. Bonolo, M. F. Lima-Costa, M. G. Medina, E. Mota, V. B de Oliveira y M. A. Turci (2010). "Major Expansion of Primary Care in Brazil Linked to Decline in Unnecessary Hospitalization". Health Affairs 29, 12: 2.149-2.160. 
Ministerio de Salud, Chile (2007). "Informe Final Estudio de Carga de Enfermedad y Carga Atribuible, Chile 2007”. http://epi.minsal.cl/epi/ html/invest/cargaenf2008/Informe\%20final\%20carga_Enf_2007.pdf.

(2011)."Encuesta Nacional de Salud Chile 2009-2010". http:// www.encuestasalud.cl/ens/wp-content/uploads/2011/09/ InformeENS_2009-2010_CAP1.pdf.

- Departamento de Estadísticas en Información en Salud (2012a). "Serie de defunciones por tumores malignos, según sexo. Chile 20002009". http://deis.minsal.cl/vitales/defunciones_serie/Defunciones Mortalidad_Tumores_Malignos_2000-2009.htm.

- (2012b). Anuario de Estadísticas de Atenciones y Recursos para la Salud, años 2005 a 2009 http://intradeis.minsal.cl/reportesrem/AtAmb_ Recursos/Anuarios/2005_2009/Anuario_2005_2009.pdf.

_ (2012c). "Atenciones de Urgencia y Hospitalizaciones Diarias, según Causa y Grupo de Edad 2008-2012". http://intradeis.minsal.cl/intradeis/ ATENCIONES_URGENCIA/Reportes/generador.aspx.

Miyamoto, A., S. Kuriyama y Y. Nishino (2007). "Lower Risk of Death from Gastric Cancer among Participants of Gastric Cancer Screening in Japan: A Population-Based Study". Preventive Medicine 44. 12-19.

Montero, J., A. Téllez y C. Herrera (2010). "Reforma Sanitaria Chilena y la Atención Primaria en Salud. Aspectos Críticos: Temas de la Agenda Pública". Centro de Políticas Públicas UC 5, 38: 1-12.

Organización Mundial de la Salud (2008). "La Atención Primaria de Salud, Más Necesaria que Nunca". Washington, DC: OMS http:/www.who.int/ whr/2008/summary/es/index.html.

Pasarín, M. I., S. Berra, L. Rajmild, M. Solans, C. Borrella y B. Starfield (2007). "Un Instrumento para la Evaluación de la Atención Primaria de Salud desde la Perspectiva de la Población”. Atención Primaria 3, 8: 395-403.

Peñaloza, B., Th. Leisewitz, L. Nogueira, G. Bastías y L. Villarroel (2008). "Evaluación del Modelo de Salud Familiar en Atención Primaria desde la Perspectiva de los Usuarios". IV Concurso del Fondo Nacional de Investigación y Desarrollo en Salud (FONIS). SA07I20032, 2007-2008.

Phillips, R. L., M. S. Dodoo y L. A Green (2009). "Usual Source of Care: An Important Source of Variation in Health Care Spending". Health Affairs 28: 567-577.

Probst, J. C., J. N. Laditka y S. B. Laditka (2009). “Association between Community Health Center and Rural Health Clinic Presence and CountyLevel Hospitalization Rates for Ambulatory Care Sensitive Conditions: An Analysis across Eight US States". Biomedcentral Health Services Research 9: 134-145.

Puschel, K., S. Sullivan, S. J. Montero, B. Thompson y A. Díaz A. (2002). "Análisis de Costo-Efectividad de un Programa Preventivo de Enfermedad Vesicular en Chile". Revista Médica de Chile 130, 4: 447-459. http://www.scielo.cl/scielo.php?pid=S003498872002000400014\&script=sci_arttext - af2.

Román, O. (2011). "Resolución Clínica en la Atención Primaria de Salud". Revista Médica de Chile 139, 9: 1.240-1.241.

Rosano, A., L. Abo, R. Falvo, J. Van der Zee, W. Ricciardi, G. Guasticch y A. G. de Belvis (2012). "The Relationship between Avoidable 
Hospitalization and Accessibility to Primary Care: A Systematic Review". European Journal of Public Health, May 29 [publicación electrónica previa a la publicación en papel].

Rosenberg, C. N., P. Peele, D. Keyser, S. McAnallen y D. Holder (2012). "Results from a Patient-Center Medical Home Pilot at UPMC Health Plan Hold Lessons for Broader Adoption of the Model". Health Affairs 31, 11: 2.423-2.431.

Serra, M., A. Arévalo, C. Ortega, A. Ripoll y N. Gimenez (2010). "Minor Surgery Activity in Primary Care". Journal of the Royal Society of Medicine: Short Reports. 1, 4: 36-44.

Servicio de Salud Metropolitano Sur Oriente. "Cuenta Pública 2010". http://www.ssmso.cl/TMPARCHIVOS/comunicaciones/ CUENTAPUBLICA2010.pdf.

Shi, L., J. Macinko, B. Starfield, J. Wulu, J. Regan y R. Politzer (2003). "The Relationship between Primary Care, Income Inequality, and Mortality in US States, 1980-1995". Journal of American Board of Family Practice 16, 5: 412-422.

Sicras-Mainar, A., J. Serrat-Tarrés, R. Navarro-Artieda, R. Llausí-Sellés, I. Ruano-Ruano, J. A. González-Ares (2007). “Adjusted Clinical Groups Use as a Measure of the Referrals Efficiency from Primary Care to Specialized in Spain". European Journal of Public Health 6: 657-663.

Simms, C y M. Rowson (2003). "Reassessment of Health Effects of the Indonesian Economic Crisis: Donors versus the Data". Lancet 361: 1.382-1.385.

Sistema Nacional de Administración Municipal (SINIM), 2010. http://www. sinim.gov.cl/indicadores/municipio).

Soto, R. (2003). "Modelo de Atención Primaria y Perspectivas". Conferencia pronunciada en el Departamento de Atención Primaria, División de Gestión de Red de Servicios, Ministerio de Salud de Chile, 14 de mayo de 2003.

Stange, K. (2009). "The Problem of Fragmentation and the Need for Integrative Solutions". Annals of Family Medicine 7, 2: 100-103.

Starfield, B. (2012). "Primary Care: An Increasingly Important Contributor to Effectiveness, Equity, and Efficiency of Health Services. SESPAS report 2012”. Gaceta Sanitaria 26 (S): 20-26.

Starfield, B. y L. Shi (2002). "Policy Relevant Determinants of Health: An International Perspective". Health Policy 60: 201-218.

Starfield, B., L. Shi y J. Macinko (2005). "Contribution of Primary Care to Health Systems and Health". Milbank Quarterly 83: 457-502.

Stigler, F., B. Starfield, M. Sprenger, H. Salzer, S. M. Campbell (2012). "Assessing Primary Care in Austria: Room for Improvement." Family Practice Nov. 11 [publicación electrónica previa a la publicación en papel]. 